Check for updates

Cite this: Mater. Chem. Front., 2020, 4, 2006

Received 17th February 2020,

Accepted 1st May 2020

DOI: 10.1039/d0qm00087f

rsc.li/frontiers-materials

\title{
Linking triptycene to silole: a fruitful association $\uparrow+$
}

\author{
Kassem Amro, ${ }^{a}$ Anil K. Thakur, ${ }^{\mathrm{b}}$ Marc Rolland, ${ }^{\mathrm{c}}$ Arie Van Der Lee, (D) ${ }^{\mathrm{c}}$ \\ Vincent Lemaur, (D) ${ }^{d}$ Roberto Lazzaroni, (D) d Joëlle Rault-Berthelot, ${ }^{e}$ Cyril Poriel, (D) e \\ Lionel Hirsch, (D) ${ }^{b}$ Sébastien Clément ${ }^{* a}$ and Philippe Gerbier (D) *a
}

\begin{abstract}
1,1'-Disubstituted-2,3,4,5-tetraphenylsiloles incorporating triptycene moieties Tp-DMTPS and Tp-MPTPS were prepared and characterized. Like other 2,3,4,5-tetraphenylsiloles, both compounds exhibit aggregation-induced emission (AIE) behavior. Unexpectedly, the solid state PL emission of Tp-DMTPS show dependence according to the degree of crystallinity of the powders. While precipitates display a blue-green emission, microcrystalline powders emit in the yellow-orange region. Moreover a rare reversible mechanohypsochromic phenomenom is observed: upon grinding, the emission of a microcrystalline powder changes from yellow-orange to blue-green, the original emission being restored by annealing the sample. The mechanism of this blue-shift upon crystal grinding was investigated by $\mathrm{X}$-ray diffraction, ${ }^{13} \mathrm{C}$ CP-MAS NMR and DFT calculations. Under mechanical stress, strong intermolecular $\mathrm{CH} / \pi$ interactions are found to promote the less energetically stable population of conformers present in the crystals, leading to this blue-shift. Finally the influence of the triptycene moieties on the thermal and structural stability of Tp-DMTPS and Tp-MPTPS was studied. Compared with DMTPS and MPTPS parents, a general enhancement of the properties is noticed, especially when used as active layer in OLED fabrication. Our results suggest that the attachment of triptycene moieties to $\pi$-conjugated molecules is an interesting way to improve their thermal, structural and photophysical properties for sensing or optoelectronic applications.
\end{abstract}

\section{Introduction}

Limited to very few applications for a long time because of their complicated synthetic chemistry, ${ }^{1}$ siloles or silacyclopentadienes experienced a spectacular rebirth thanks to the works of K. Tamao and S. Yamaguchi in the late 90 s. $^{2,3}$ Indeed, the general and versatile synthesis via an intramolecular reductive cyclization of diethynylsilanes they proposed allowed the preparation of various 2,5-difunctional siloles that would be

\footnotetext{
${ }^{a}$ Institut Charles Gerhardt de Montpellier (ICGM, UMR 5253), Université de Montpellier, CNRS, ENSCM, F-34095 Montpellier, France.

E-mail: philippe.gerbier@umontpellier.fr, sebastien.clement1@umontpellier.fr

${ }^{b}$ Institut Européen des Membranes (IEM, UMR 5635), Université de Montpellier, CNRS, ENSCM, F-34095 Montpellier, France

${ }^{c}$ Laboratoire de l'Intégration du Matériau au Système (IMS, UMR 5218), Université de Bordeaux, CNRS, Bordeaux INP, ENSCBP, 33405 Talence, France

${ }^{d}$ Laboratoire de Chimie des Matériaux Nouveaux, Institut de Recherche sur les Matériaux, Université de Mons (UMONS), 7000 Mons, Belgium

${ }^{e}$ Institut des Sciences Chimiques de Rennes, (ISCR, UMR 6226),

Université de Rennes, CNRS, F-35000 Rennes, France

$\dagger$ Dedicated to Profs J. Veciana and C. Rovira at the occasion of their 70th anniversary.

\# Electronic supplementary information (ESI) available. CCDC 1963525 and 1963526. For ESI and crystallographic data in CIF or other electronic format see DOI: 10.1039/ d0qm00087f
}

otherwise very difficult to obtain. Shortly afterwards, on account of their unique electronic properties, i.e., their very low LUMO energy levels combined with high fluorescence quantum yields in the solid state, siloles were used successfully as both emissive and electron-transporting materials in organic light emitting diodes (OLED) manufacture. ${ }^{4-7}$ The origin of their electronic properties is the consequence of an efficient interaction between the $\sigma^{*}$ orbital of the silole silicon atom with the $\pi^{*}$ orbital of the butadiene fragment ${ }^{5,8}$ whereas the origin of their outstanding high solid-state fluorescence is attributed to the aggregation induced emission (AIE) phenomenon that was described for the first time with 1-methyl-1,2,3,4,5-pentaphenylsilole. ${ }^{9}$ Since then, AIE materials are the subject of intense scientific research and have found many applications that encompass various domains going from organic electronics to theranostic bioprobes. ${ }^{10-14}$ Moreover, with the growing number of AIE materials described in the literature, rather new and uncommon phenomena such as reversible mechanofluorochromism have started to be reported since their first observation with tetraphenylethene (TPE) and diphenyldibenzofulvene (DPDBF) derivatives. ${ }^{15-20}$

In small molecules-based organic electronics, besides the device efficiency, the thermal and structural stabilities of the used organic materials are an important concern. ${ }^{21,22}$ Indeed, for instance, an operating OLED releases heat that may alter 


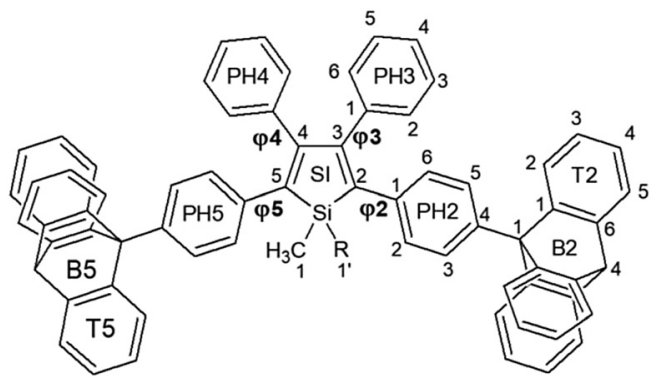

Fig. 1 Labelling scheme for Tp-DMTPS $\left(R 1^{\prime}=\right.$ Me) and Tp-MPTPS $\left(\mathrm{R} 1^{\prime}=\mathrm{Ph}\right)$.

quickly its efficiency. ${ }^{23}$ This is the reason why materials with high melting points and high glass transition temperatures $\left(T_{\mathrm{g}}\right)$ are desirable to achieve the fabrication of devices with good durability. One of the strategies used to increase the thermal stability of the organic layers is to crosslink the adequately functionalized molecules after their deposition. ${ }^{24}$ Another strategy involves the control of the spatial organization, i.e., distance and relative orientation of the constitutive molecules through the chemical synthesis and/or the use of an appropriate construction scaffold. ${ }^{25}$ Triptycene with its fascinating paddlewheel shape has been widely used for this purpose in supramolecular chemistry, polymer chemistry and materials science. ${ }^{26-28}$ However, in spite of the thermal stabilization afforded by its presence in the molecular structures, only few examples of the use triptycene-based luminescent materials in OLEDs have been reported so far. ${ }^{29-31}$

With this in mind we report herein the synthesis and structural characterization of two novel silole derivatives incorporating triptycene moieties at the 2,5-positions (Tp-DMTPS and Tp-MPTPS, Fig. 1) with the first aim to use them as emissive layers in OLEDs. We will focus on the interplay between the triptycene side-groups and the silole core that induces an original behavior in terms of thermal and structural stability, luminescence and mechanofluorohypsochromism, phenomenon that was still rarely observed up to now. ${ }^{32-37}$ We show that the modulation of the structure in the solid state resulting from the incorporation of triptycene groups affords an enhancement of OLED performance by formation of morphologically stable non-crystalline emissive layers.

\section{Results and discussion}

\section{Synthesis}

The siloles Tp-DMTPS and Tp-MPTPS were conveniently prepared by the method previously described by Tamao et al. that involves the intramolecular reductive cyclization of 1,1-bis(phenylethynyl)silane, followed by a palladium-catalyzed cross-coupling reaction with the appropriate bromoaryl compound (Scheme 1). ${ }^{4,38}$ 9 -( $p$-Bromophenyltriptycene) was prepared via a Diels-Alder reaction between in situ generated benzyne, and $4^{\prime}$-bromophenylanthracene. ${ }^{39}$ Tp-DMTPS and Tp-MPTPS were isolated in 54 and $49 \%$ yields, respectively, as pale yellow powders.

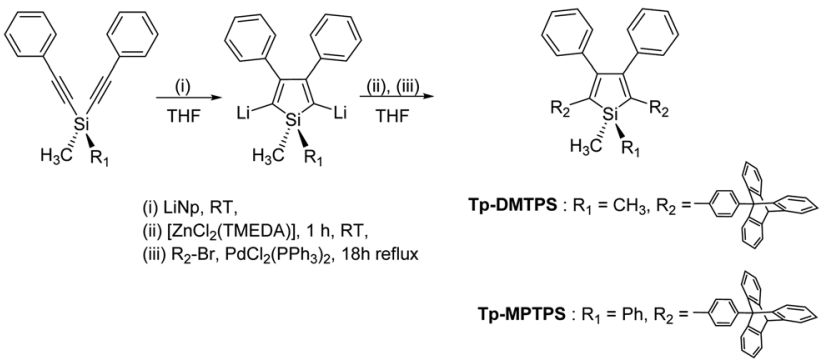

Scheme 1 Synthetic route to Tp-DMTPS and Tp-MPTPS (see more details in ESI\$).

\section{Crystal structure}

As shown in Fig. 2 (and Fig. S3-S6, ESI $\$$ ), the molecular packing of Tp-DMTPS is mainly governed by the paving of the triptycene moieties in alternating up (one of the phenyl ring parallel to the $c$-axis) and down (antiparallel to the $c$-axis) columns leading to a layered structure. Inside the triptycene double layer, the 3,4diphenylsilole moieties also adopt an up and down columnar packing corresponding to the orientation of the triptycene sidegroups. Interestingly, the structure shows that the silole rings could be inclined either on the right or on the left with respect to a plane going through the middle of a column (Fig. 3). This situation leads to the co-existence of two conformers in the crystal, which will be named $R$ and $L$. In the $R$-conformer, the methyl group C69 (see Fig. 3) is nestled between two phenyl
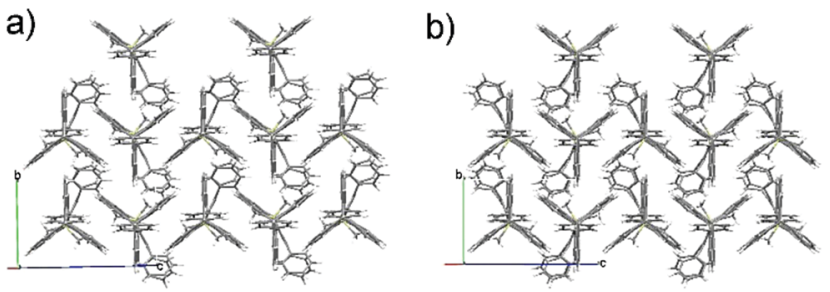

Fig. 2 Views of the crystal packing of Tp-DMTPS showing the triptycene paving in the bc plane for (a) $R$-conformers and (b) $L$-conformers.

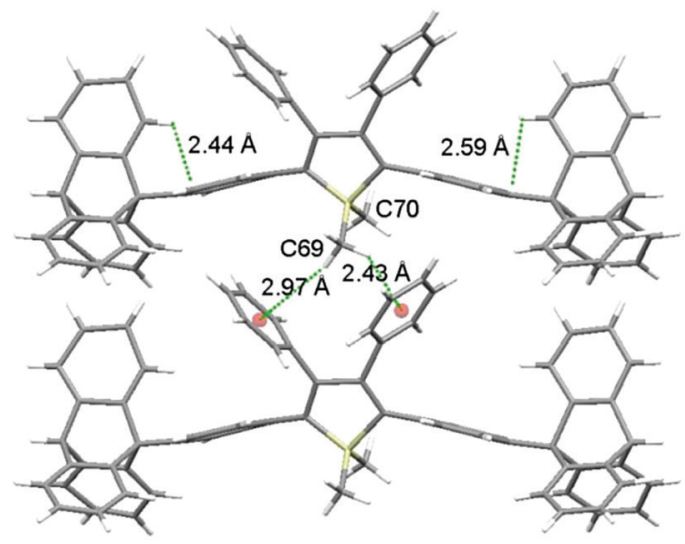

Fig. 3 View of the $R$-conformers of Tp-DMTPS in the ab plane showing the methyl C69 nestling between the aromatic rings. Intra and intermolecular $\mathrm{CH} / \pi$ interactions are marked with green dotted lines. 
rings of the molecule immediately below. Short $\mathrm{CH} / \pi$ hydrogen bonds are present between two hydrogen atoms belonging to the methyl group and the interacting phenyl rings $\left(d_{\mathrm{CH} / \pi}=\right.$ $2.43 \AA$ and $2.97 \AA$ ). ${ }^{40}$ In the $L$-conformer (Fig. S5 and S6, ESI $\$$ ), the other methyl group $\mathrm{C} 70$ is nestled between the phenyl rings and shorter $\mathrm{CH} / \pi$ hydrogen bonds are observed $\left(d_{\mathrm{CH} / \pi}=2.55 \AA\right.$ and $2.85 \AA$ ).

The distance and the orientation of the $\mathrm{CH} / \pi$ hydrogen bonds observed in each conformer are in the range of what is frequently observed in the literature $\left(d_{\mathrm{CH} / \pi}=2.73 \pm 0.13 \AA\right) .{ }^{40}$ Since it is only the 1,1-dimethyl-3,4-diphenylsilole moiety that is involved in this swinging, this induces torsion angle modifications between the silole core and the four phenyl rings at its periphery (Table 1). The phenyl rings located at the 2,5position of the silole core are locked within the structure because of a strong $\mathrm{CH} / \pi$ interaction between their aromatic system and a hydrogen atom belonging to the triptycene moieties $\left(d_{\mathrm{CH} / \pi}=2.44 \AA\right.$ and $\left.2.59 \AA\right)$. This $\mathrm{CH} / \pi$ interaction leads to a moderate bending of the phenyl moiety with respect to the threefold axis of the triptycene by $\mathrm{ca} .17^{\circ}$ (a bending angle of $6^{\circ}$ is found in the crystal structure of 9-phenyltriptycene ${ }^{41}$ ). If present in solution, this strong $\mathrm{CH} / \pi$ interaction should lead to an appreciable shift of the ${ }^{1} \mathrm{H}$ NMR signal of the corresponding hydrogen atom compared to the two other ones, because of the shielding anisotropy cones of the adjacent phenyl ring. ${ }^{42}$ However in none of the molecules incorporating triptycene moieties we have synthesized (see Fig. S30-S32, ESI $\$$ ), this feature was observed. In every cases, the hydrogen atoms of the $\mathrm{C}(2)$ carbon atoms (see Fig. 1 for labelling) belonging to the triptycene paddlewheels are equivalents, indicating a free rotation of the later in solution at ambient temperature.

The crystal packing of Tp-MPTPS (Fig. S7, ESI $\ddagger$ ) does not show any of the features found with Tp-DMTPS. Probably because of the presence of a phenyl ring attached to the silicon atom, the triptycene moieties are not able to organize themselves in regular layers. Consequently, the molecular packing is less dense in Tp-MPTPS and $\mathrm{CH}_{2} \mathrm{Cl}_{2}$ molecules are found in the structural voids. Notably short $\mathrm{CCl} / \pi$ halogen interactions are also observed between one chlorine atom belonging to these molecules and one of the phenyl rings adjacent to the silole ring $\left(d_{\mathrm{CCl} / \pi}=3.51 \AA\right.$, see Fig. S2, ESI $\$){ }^{43}$ Because of the chirality of the silicon atom in Tp-MPTPS, both $(R)$ and $(S)$ enantiomers were found in the crystal lattice. The molecular structure of Tp-DMTPS shows a paddlewheel arrangement of the 2, 3, 4 and 5-phenyl rings around the

Table 1 Selected torsion angles and calculated orbital energies of Tp-DMTPS and Tp-MPTPS

\begin{tabular}{|c|c|c|c|c|c|c|c|c|}
\hline & & $\begin{array}{l}\varphi_{2} \\
\left(^{\circ}\right)\end{array}$ & $\begin{array}{l}\varphi_{3} \\
\left(^{\circ}\right)\end{array}$ & $\begin{array}{l}\varphi_{4} \\
\left(^{\circ}\right)\end{array}$ & $\begin{array}{l}\varphi_{5} \\
\left(^{\circ}\right)\end{array}$ & $\begin{array}{l}\text { HOMO } \\
(\mathrm{eV})\end{array}$ & $\begin{array}{l}\text { LUMO } \\
(\mathrm{eV})\end{array}$ & $\begin{array}{l}E_{\mathrm{g}} \\
(\mathrm{eV})\end{array}$ \\
\hline \multirow[t]{3}{*}{$\overline{\text { Tp-DMTPS }}$} & Optimized $^{a}$ & 42.6 & 59.5 & 58.2 & 42.6 & -5.56 & -1.99 & 3.57 \\
\hline & $R$-conformer ${ }^{b}$ & 54.0 & 70.2 & 50.4 & 58.6 & -5.65 & -1.81 & 3.84 \\
\hline & $L$-conformer ${ }^{b}$ & 74.2 & 76.0 & 71.4 & 67.5 & -5.88 & -1.64 & 4.24 \\
\hline \multirow[t]{2}{*}{ Tp-MPTPS } & Optimized $^{a}$ & 43.9 & 59.4 & 58.1 & 43.6 & -5.58 & -2.00 & 3.58 \\
\hline & X-ray & 51.4 & 53.6 & 56.2 & 33.1 & & & \\
\hline
\end{tabular}

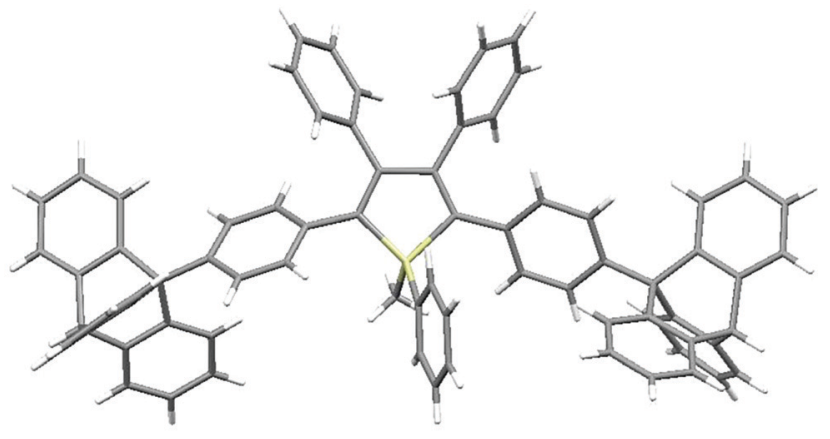

Fig. 4 View of Tp-MPTPS molecular structure.

silole core, whereas a more conventional propeller-like disposition of the phenyl rings is found in Tp-MPTPS (Fig. 4). In this case, the structure also reveals that the phenyl moieties are bent off the threefold axis of the triptycene by angle of $16^{\circ}$ on average.

\section{Hirshfeld surface analysis}

The Hirshfeld surfaces ${ }^{44}$ of both conformers of Tp-DMTPS (Fig. 5) were calculated with the CrystalExplorer $\operatorname{program}^{45}$ and are mapped with the $d_{\text {norm }}$ property ${ }^{46}$ where the color scheme goes from red $\left(d_{\text {norm }}=-0.2664\right.$ and -0.2795 for conformers $L$ - and $R$-, respectively) to blue $\left(d_{\text {norm }}=2.2715\right.$ and 2.2586 for conformers $L$ - and $R$-, respectively). The most notable interactions shown in red in Fig. 5 are indeed in both cases between the methyl hydrogens and the aromatic rings of adjacent molecules. Interestingly, in $R$-conformer, the secondary interactions between the $\mathrm{CH}$ systems of one ring to the $\pi$-system of an adjacent ring seem to be less important than in $L$-conformer. An analysis of the elemental fingerprint plots shows that the relative contributions of the different interactions in the two conformers are anyhow approximately equal: $31.7 \% / 31.3 \%$ for $\mathrm{H} \cdots \mathrm{C} / \mathrm{C} \cdots \mathrm{H}$ interactions and $64.8 / 65.2 \%$ for hydrophobic $\mathrm{H} \cdots \mathrm{H}$ interactions. The Hirshfeld surface analysis of Tp-MPTPS (Fig. S8, ESI ) shows $d_{\text {norm }}$ ranging from -0.1149 to 2.3526 with
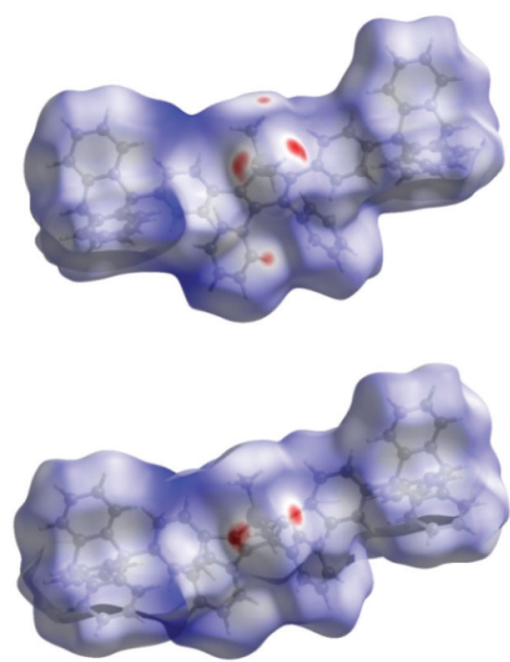

Fig. 5 Hirshfeld surfaces for the $L$-conformer (top) and the $R$-conformer (bottom) of Tp-DMTPS. 
the following relative elemental interactions: $\mathrm{H} \cdots \mathrm{H}(62.4 \%), \mathrm{H} \cdots \mathrm{C}$ $(31.5 \%), \mathrm{H} \cdots \mathrm{Cl}(3.6 \%)$ and $\mathrm{C} \cdots \mathrm{Cl}(0.7 \%)$. However, it is clearly seen that the intermolecular interactions in the case of Tp-MPTPS are more dispersive than in the case of Tp-DMTPS.

\section{Thermal properties}

The thermal properties of Tp-DMTPS and Tp-MPTPS were examined by thermogravimetric analysis (TGA) and differential scanning calorimetry (DSC) measurements. As shown in Fig. 6, the TGA of Tp-DMTPS exhibits a weight loss in one step leading to a high decomposition temperature $\left(T_{\mathrm{d}}\right)$ of $486^{\circ} \mathrm{C}$. In contrast, the weight loss of Tp-MPTPS takes place in two steps. The first weight loss of $c a .10 \%$ could be ascribed to the loss of interstitial solvent molecules, as observed in the crystal structure of Tp-MPTPS (see above). This high temperature of desolvation may be due to the strong $\mathrm{CCl} / \pi$ halogen interactions that have been characterized in the crystal lattice. The second weight loss is due to the decomposition of Tp-MPTPS molecule. As for Tp-DMTPS, a high $T_{\mathrm{d}}$ of $489{ }^{\circ} \mathrm{C}$ was noticed highlighting the high thermal stability of these two siloles. It is noteworthy that these $T_{\mathrm{d}}$ are higher than their parent compounds, namely, 1,1-dimethyl-2,3,4,5-tetraphenylsilole (DMTPS): $272{ }^{\circ} \mathrm{C}$ and 1-methyl-1,2,3,4,5-pentaphenylsilole (MPTPS): $309{ }^{\circ} \mathrm{C}^{47}$ The same trend is observed with the melting point, measured at $431{ }^{\circ} \mathrm{C}$ for Tp-DMTPS; $250{ }^{\circ} \mathrm{C}$ above that of the DMTPS parent (Fig. S13, ESI $)^{47}$

\section{Photophysical properties}

The UV-visible absorption and fluorescence spectra were measured in dilute dichloromethane solution (Fig. 7). The data are gathered in Table 2. Tp-DMTPS exhibits an absorption maximum at $357 \mathrm{~nm}$ corresponding to the $\pi-\pi^{*}$ transition involving mainly the HOMO and the LUMO energy levels of the silole core (see below and Fig. 13). ${ }^{48}$ For Tp-MPTPS this transition is slightly red-shifted at $369 \mathrm{~nm}$. This shift could be explained by the stabilization effect of the LUMO induced by the presence of the phenyl group on the silicon atom. ${ }^{5}$ The absorption maximum of thin films is

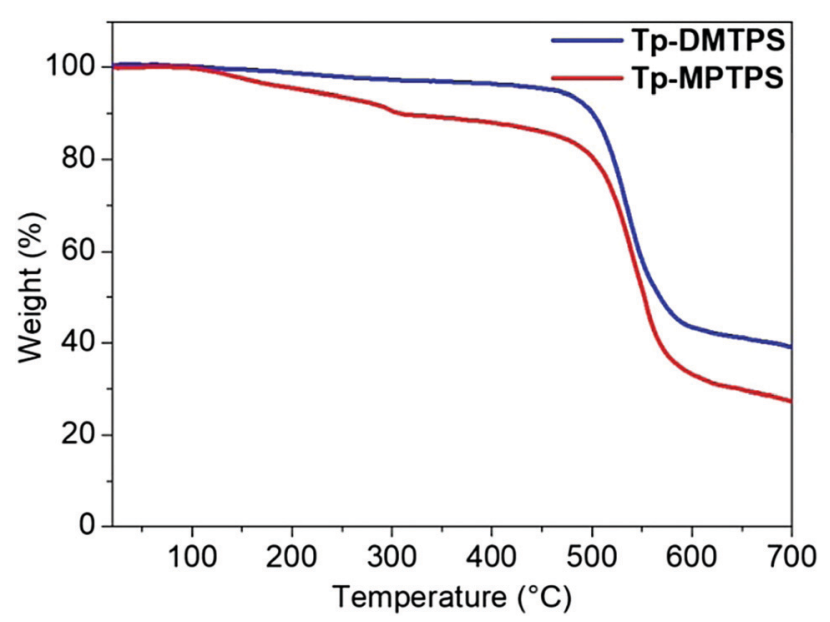

Fig. 6 TGA plots of Tp-DMTPS and Tp-MPTPS under nitrogen at a heating rate of $10{ }^{\circ} \mathrm{C} \min ^{-1}$.

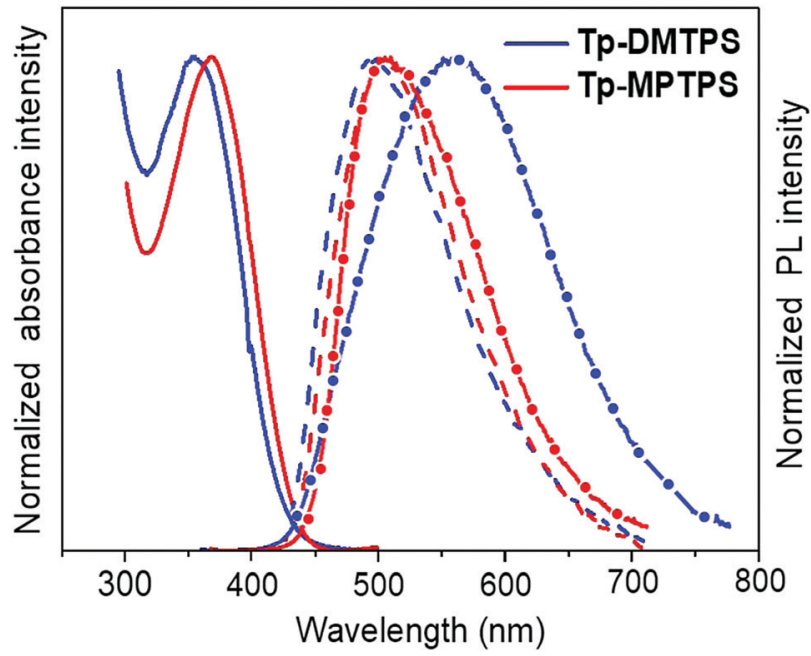

Fig. 7 Absorption (solid lines) and photoluminescence (PL) normalized spectra of Tp-DMTPS and Tp-MPTPS in $\mathrm{CH}_{2} \mathrm{Cl}_{2}$ solution $\left(10^{-5} \mathrm{M}\right.$, dashed lines) and as microcrystalline powders (lines and dotted lines).

slightly red-shifted to $365 \mathrm{~nm}$ for Tp-DMTPS (Fig. S9, ESI $\ddagger$ ), but remains unchanged for Tp-MPTPS.

The photoluminescence spectra in solution show faint PL signals with maxima at $486 \mathrm{~nm}\left(\Phi_{\mathrm{f}}=1.4 \%\right)$ and $491 \mathrm{~nm}\left(\Phi_{\mathrm{f}}=\right.$ 1.2\%) for Tp-DMTPS and Tp-MPTPS, respectively (Fig. 7). In comparison with the corresponding siloles without triptycene, DMTPS $\left(\lambda_{\text {max }}^{\mathrm{f}}=470 \mathrm{~nm}, \Phi_{\mathrm{f}}=0.2 \%\right)$ and MPTPS $\left(\lambda_{\max }^{\mathrm{f}}=471 \mathrm{~nm}\right.$, $\left.\Phi_{\mathrm{f}}=0.1 \%\right),{ }^{47}$ Tp-DMTPS and Tp-MPTPS both shows a small red shift in the emission maxima and a much higher quantum yield.

Since it is known that it is the intramolecular rotation of the phenyl rings in 2,3,4,5-tetraphenylsiloles that makes them essentially non-luminescent when molecularly dissolved, ${ }^{49}$ the enhancement of the quantum yields is probably due to the restriction of such intramolecular rotation (RIR) afforded by the triptycene moieties.

In contrast to what is observed in solution, the microcrystalline powders of Tp-DMTPS and Tp-MPTPS display a rather good fluorescence with emission maxima at 561 and $498 \mathrm{~nm}$, and quantum yields of 43 and $24 \%$, respectively. The same trend is also observed with DMTPS and MPTPS and was related to the aggregation induced emission (AIE) phenomenon that was reported for the first time with hexaphenylsilole. ${ }^{50}$ However, the significant red-shift found in the Tp-DMTPS PL spectrum in comparison with what was observed both in solution $\left(\lambda_{\max }=\right.$ $486 \mathrm{~nm})$ or deposited as $100 \mathrm{~nm}$ thick films $\left(\lambda_{\max }=485 \mathrm{~nm}\right.$, Fig. S10, ESI ) is surprising.

\section{Aggregation induced emission (AIE)}

The AIE properties of Tp-DMTPS and Tp-MPTPS were studied in THF/water mixtures with different water fractions $\left(f_{\mathrm{w}}\right)$ in view of fine-tuning the aggregation extent (Fig. 8). As expected, the larger the water fraction, the stronger the fluorescence; molecular aggregation inducing a red shift of $12 \mathrm{~nm}$ in the emission spectrum. As shown in Fig. 8, when $f_{\mathrm{w}}$ varies from 0 to $60 \mathrm{vol} \%$, the fluorescence of Tp-DMTPS remains very low. A remarkable 50 -fold increase of fluorescence intensity is observed at a water fraction of 90 vol\%. The same trend is observed with Tp-MPTPS 
Table 2 Optical and electronic properties of Tp-DMTPS and Tp-MPTPS ${ }^{a}$

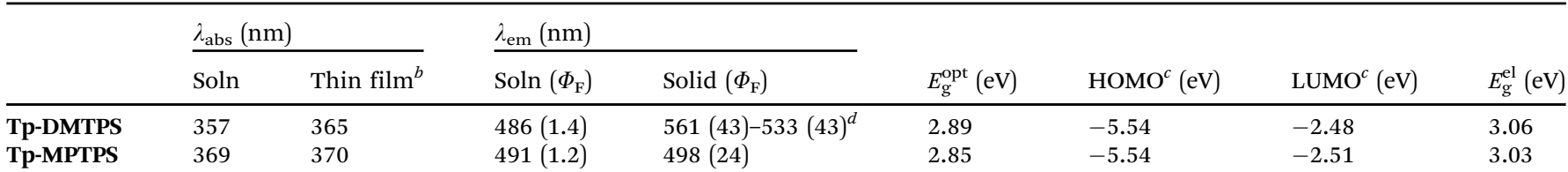

${ }^{a} \lambda_{\mathrm{abs}}=$ absorption maximum; $\lambda_{\mathrm{em}}=$ emission maximum; $\Phi_{\mathrm{F}}=$ fluorescence quantum yield in $\mathrm{CH}_{2} \mathrm{Cl}_{2}$ solutions (Soln) and in the solid state (solid); $E_{\mathrm{g}}^{\mathrm{opt}}=$ energy gap calculated from the absorption edge; $E_{\mathrm{g}}^{\mathrm{el}}=$ electrochemical gap obtained from HOMO and LUMO. ${ }^{b} 100 \mathrm{~nm}$ thick film. ${ }^{c}$ HOMO and LUMO levels determined from CVs in $\mathrm{CH}_{2} \mathrm{Cl}_{2} / \mathrm{Bu}_{4} \mathrm{NPF}_{6}{ }^{d}$ First values: microcrystalline powders, second values: ground powders. Excitation wavelength: $365 \mathrm{~nm}$.
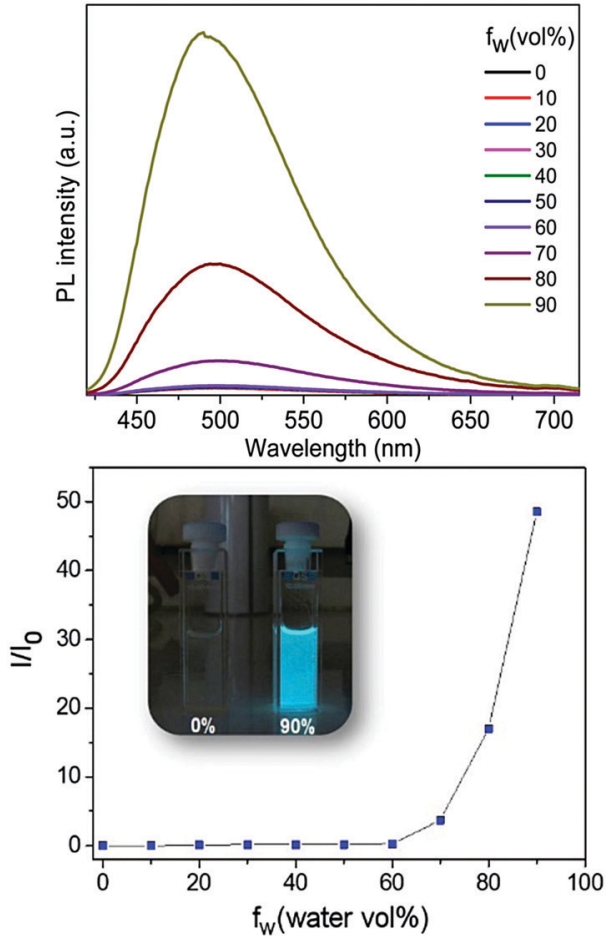

Fig. 8 Top: PL spectra of Tp-DMTPS in THF/water mixtures with different water fractions $\left(f_{\mathrm{w}}\right)$. Concentration: $10^{-5} \mathrm{M}$; excitation wavelength: $365 \mathrm{~nm}$. Bottom: Plot of $\left(I / I_{0}\right)$ values versus water fractions in THF/water mixtures. $I_{0}$ is the PL intensity in pure THF solution. Inset: Photographs Tp-DMTPS in THF/water mixtures $\left(f_{\mathrm{w}}=0\right.$ and $90 \%$ ) taken under UV lamp illumination.

with a 60 -fold increase of the fluorescence intensity when $f_{\mathrm{w}}$ also reached $90 \%$ (Fig. S12, ESI $\ddagger$ ).

Moreover, we found that unlike Tp-MPTPS, the position of the emission of Tp-DMTPS in the solid state depends strongly on its degree of crystallization. Whereas rapid precipitation of Tp-DMTPS by addition of pentane affords a solid exhibiting blue emission under excitation at $365 \mathrm{~nm}$, slow crystallization from acetone/dichloromethane mixtures leads to a yelloworange emission for the obtained microcrystals (Fig. S11, ESI $\ddagger$ ). These changes in emission features should mainly originate from the transition from the crystal to the amorphous state as well as the changes in the molecular conformations. ${ }^{50-55}$

\section{Mechanofluorochromism}

The dependence of the position of the Tp-DMTPS emission maximum on its procedure of isolation (precipitation/crystallization),

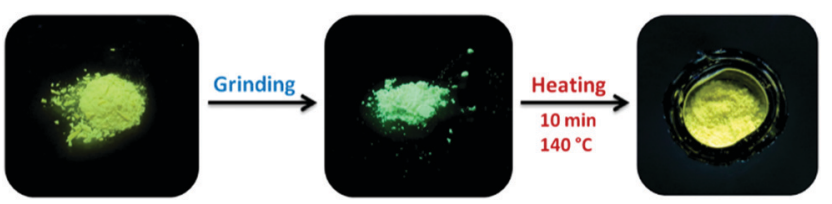

Fig. 9 Photographs of Tp-DMTPS under UV illumination before grinding (left), after grinding (middle) and after annealing at $140{ }^{\circ} \mathrm{C}$ for $10 \mathrm{~min}$ (right).

suggests that this compound could display mechanofluorochromism. ${ }^{18,56,57}$ This hypothesis was confirmed by simply grinding a sample of microcrystalline Tp-DMTPS. As shown in Fig. 9, the emission turned from orange to blue-green upon UV illumination. It is important to mention that this phenomenon called mechanohypsochromism is rarely observed, a red-shift being observed in almost all cases. ${ }^{32-37}$ Finally, the initial orange emission could be restored by heating the ground solid at $140{ }^{\circ} \mathrm{C}$ for $10 \mathrm{~min}$. The choice of this annealing temperature was based on the DSC analyses of Tp-DMTPS after grinding, which shows an exothermic signal associated to a crystallization process (Fig. S13, ESI $\$$ ).

The corresponding PL spectra (Fig. 10) shows that the grinding process is not only affecting the position of the emission maximum $\left(\lambda_{\mathrm{em}}\right.$ (crystals) $=561 \mathrm{~nm}, \lambda_{\mathrm{em}}($ after grinding $\left.)=533 \mathrm{~nm}\right)$, but is also modifying the peak shape, the solid state quantum yield of emission remaining the same. Interestingly, the emission of the aggregates generated by adding water to a THF solution shows a sharper emission peak $\left(\lambda_{\mathrm{em}}=498 \mathrm{~nm}\right)$, which is very similar to the one obtained in solution (see Fig. 7). These emission changes should mainly be due to their different molecular conformations that are affected by the crystallinity in these solids. ${ }^{54,58}$

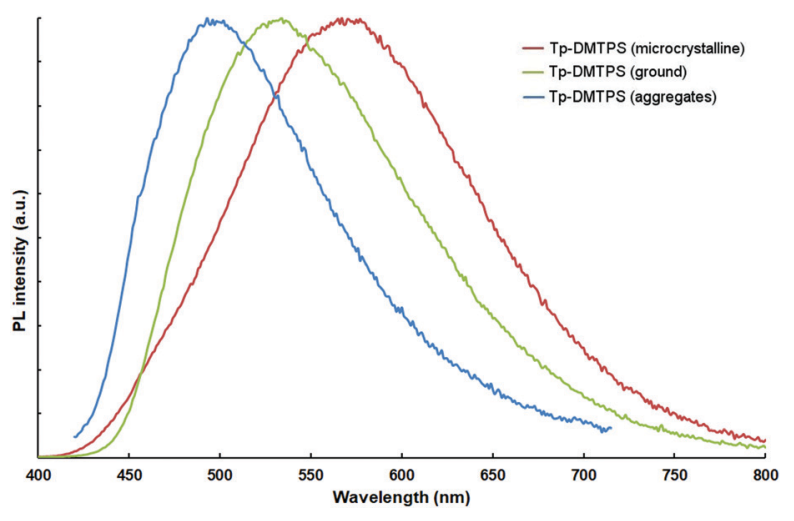

Fig. 10 PL spectra of Tp-DMTPS in various forms. 
To evaluate the degree of crystallinity, we performed powder XRD analyses, the patterns indicate that grinding microcrystalline Tp-DMTPS leads to a dramatic decrease of its crystallinity, but not reaching complete amorphization (Fig. S14, ESI $\ddagger$ ). On the other hand, fast precipitation from a mixture of THF with "high" water content should lead to the formation of amorphous aggregates, as previously observed by Tang et al. ${ }^{50,55}$ Therefore, there is a clear dependency between the long-range molecular order and the emission: the higher the order, the stronger the emission is redshifted. Interestingly, similar experiments conducted with microcrystalline Tp-MPTPS powders showed that the position of the emission maximum remains unaffected upon grinding.

\section{Solid state NMR experiments}

${ }^{13} \mathrm{C}$ CP-MAS NMR spectra of either microcrystalline or ground powders of Tp-DMTPS are shown in Fig. 11. In comparison with the spectrum recorded in $\mathrm{CDCl}_{3}$ solution, the peaks were observed at very similar chemical shifts. The signal arising from the resonance of the methyl groups $\mathrm{SI}\left(\mathrm{C} 1, \mathrm{C} 1^{\prime}\right)$ at $\delta=-3.6 \mathrm{ppm}$ is split into two, as well as the signals from the carbon atoms belonging to the silole ring $\mathrm{SI}(\mathrm{C} 3, \mathrm{C} 4) \delta=154.8 \mathrm{ppm}$ and $\operatorname{SI}(\mathrm{C} 2, \mathrm{C} 5) \delta=141.8 \mathrm{ppm}$ (see Fig. 1 for labelling). The same splitting is also observed for the carbon atoms $\mathrm{PH} 2(\mathrm{C} 1)$ and $\mathrm{PH} 5(\mathrm{C} 1)(\delta=139.0 \mathrm{ppm})$, which are directly connected to the silole ring. It is noteworthy that this peak splitting is also noticed, in the ${ }^{13} \mathrm{C}$ CP-MAS NMR spectrum of DMTPS but with a smaller extent (Fig. S15, ESI ). As shown in Fig. 11, grinding the microcrystalline powder does not affect the spectrum pattern to a large extent; only a peak broadening is observed.

These observations are in line with what has been learned from the crystal structure analysis, i.e. the splitting of the methyl group signals reflects their non-equivalent environment in the crystal lattice, one being nestled between two phenyl rings. As mentioned above, going from the $R$ - to the $L$-conformer brings modifications of the torsion angles $\varphi_{2}, \varphi_{3}, \varphi_{4}$ and $\varphi_{5}$. Because of them, the conjugation between the surrounding phenyl rings and the silole core should be affected and the resonance peaks corresponding to the carbon atoms involved in the conjugation pathway split into two. This is also what was observed for

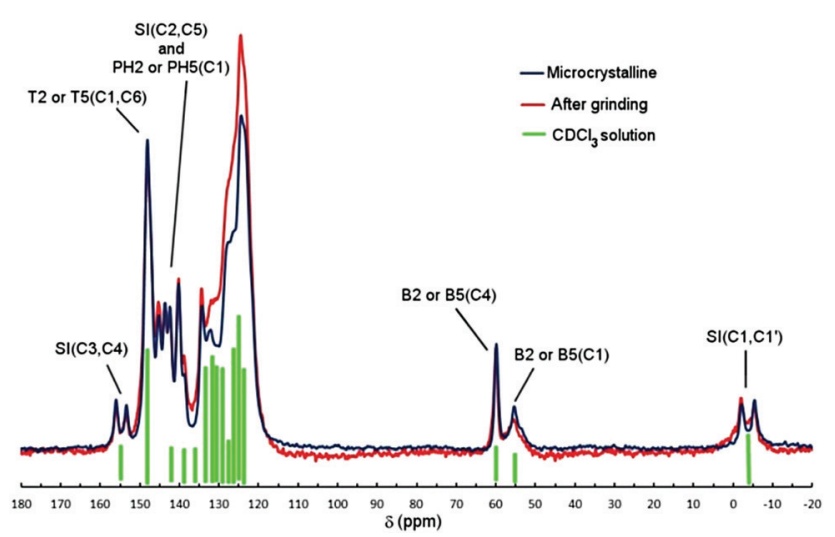

Fig. $11{ }^{13} \mathrm{C}$ CP-MAS NMR spectra of Tp-DMTPS (microcrystalline and ground). Green bars represent the peaks observed in the ${ }^{13} \mathrm{C}\left\{{ }^{1} \mathrm{H}\right\}$ NMR spectrum recorded in $\mathrm{CDCl}_{3}$ solution. See Fig. 1 for labelling.
DMTPS, its crystal structure exhibiting two molecules in the asymmetric unit that mainly differ in the rotation of the phenyl groups with respect to the central silole ring. ${ }^{47}$

From the ${ }^{13} \mathrm{C}$ CP-MAS NMR experiments, we have shown that the spectrum of the ground powder is very similar to that of the crystalline one. The only difference consists in the broadening of some resonance peaks that should originate from the loss of crystallinity, as observed by XRD. Therefore, the two methyl groups remaining non-equivalent, this should indicate that a methyl group is always nestled between two phenyl rings both in the crystalline and the ground form. Interestingly, since the emission is blue-shifted, disordering the crystal packing upon grinding appears to favour the emission originating from the less conjugated $L$-conformer. This preference should be due to the stronger $\mathrm{CH} / \pi$ interactions found with this conformer. Finally, when vacuum-deposited as thin films, the PL and EL spectra showed similarities with that measured in solution. This indicates that this deposition process does not promote a good molecular organization even at a very small scale. Contrastingly, Tp-MPTPS displayed a more conventional behavior since the conformation of the molecules do not show any noticeable change depending on their physical state as it is observed with its parent MPTPS.

\section{Electrochemical properties}

Cyclic voltammetry (CV) of Tp-DMTPS and Tp-MPTPS were recorded in $\mathrm{CH}_{2} \mathrm{Cl}_{2} / \mathrm{Bu}_{4} \mathrm{NPF}_{6}(0.2 \mathrm{M})$ both in oxidation and reduction. In oxidation (Fig. 12, up), both compounds show similar successive oxidation steps with maxima closed to 1.26 ,
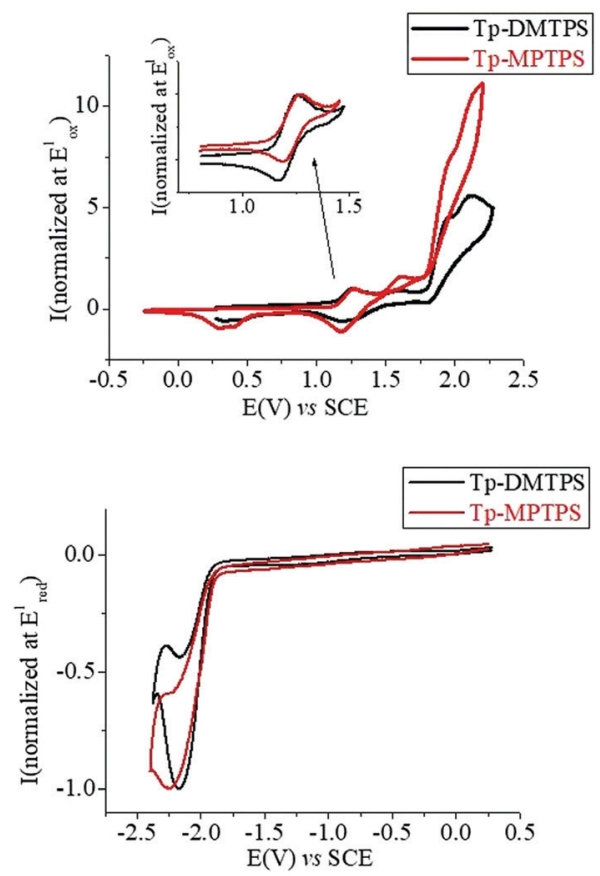

Fig. 12 Top: Cyclic voltammetry in $\mathrm{CH}_{2} \mathrm{Cl}_{2} / \mathrm{Bu}_{4} \mathrm{NPF}_{6}$ (0.2 M) of Tp-DMTPS (in black) and Tp-MPTPS (in red). Exploration in the anodic direction (top) and in the cathodic direction (bottom). Working electrode: platinum disk, sweep-rate: $100 \mathrm{mV} \mathrm{s}^{-1}$. 
$1.60,1.90$ and $2.10 \mathrm{~V}$. Only the first oxidation step is reversible for the two compounds (see inset Fig. 12, top). From the onset potential of this first oxidation ( $1.14 \mathrm{~V}$ for both compounds), the HOMO levels were calculated at $-5.54 \mathrm{eV} \cdot{ }^{59,60}$ Thus, in the light of theoretical calculation (see further, Fig. 15), this first electron transfer is centered on the phenyl/silole moiety. Recurrent cycles recorded up to the fourth oxidation step, lead for the two compounds to electrodeposition processes with the appearance and growth of new redox systems along the cycles and to the coverage of the electrode surface by insoluble electroactive deposits (Fig. S16 and S17, ESI $\$$ ). Although this observation is not directly of interest in the topic of the present work, it is worth mentioning that the deposit obtained from Tp-MPTPS present both p- and a n-doping processes with a gap as low as $1.80 \mathrm{eV}$ (see Fig. S17, ESI, $\ddagger$ right).

As electropolymerization is a useful technique to design low gap materials, ${ }^{61}$ the present silole derivatives may be investigated in the future for that purpose. In reduction, both compounds exhibit a similar irreversible reduction wave (Fig. 12, bottom) from which the LUMO levels were calculated around $-2.5 \mathrm{eV}\left(-2.48 \mathrm{eV}\right.$ for Tp-DMTPS $\left(E_{\text {onset }}^{\text {red }}-1.92 \mathrm{~V}\right)$ and $-2.51 \mathrm{eV}$ for Tp-MPTPS $\left(E_{\text {onset }}^{\text {red }}:-1.89 \mathrm{~V}\right)$ ). The electrochemical gaps $\left(E_{\mathrm{g}}^{\mathrm{el}}\right)$ for both compounds were calculated to be $3.06 \mathrm{eV}$ for Tp-DMTPS and $3.03 \mathrm{eV}$ for Tp-MPTPS. These $E_{\mathrm{g}}^{\mathrm{el}}$ values and their contraction of $0.03 \mathrm{eV}$ from Tp-DMTPS to Tp-MPTPS are in accordance with the optical gaps calculated from absorption spectra recorded in $\mathrm{CH}_{2} \mathrm{Cl}_{2}\left(E_{\mathrm{g}}^{\mathrm{el}}=2.89 \mathrm{eV}\right.$ for Tp-DMTPS and $2.85 \mathrm{eV}$ for Tp-DMTPS).

\section{Electroluminescence}

To evaluate the benefit brought by tritycene groups, we compared Tp-DMTPS, Tp-MPTPS and DMTPS as both emissive and electron transporting layer in OLEDs. Since silole derivatives are good electron transporting materials, particular efforts must be done to have efficient hole injection at the anode for getting high luminescence. ${ }^{62}$ Therefore, we have used an ITO/PEDOT-PSS/ $\mathrm{NPB} /$ silole/Ca simplified architecture to evaluate the performances of these compounds without trying to especially improve them. Because of its tendency to form highly crystalline thin films (Fig. S18, ESI ), it was very difficult to collect reproducible data from devices made with DMTPS. However, we were able to make measurements showing very low performances with a quantum efficiency of $0.08 \mathrm{~cd} \mathrm{~A}^{-1}$ at $6.0 \mathrm{~V}$ (Fig. S19, ESI $)$ which is in line with what has been previously described. ${ }^{63}$ Contrastingly, Tp-DMTPS displays good film-forming ability, the thin films remaining amorphous even after being annealed at $80{ }^{\circ} \mathrm{C}$ for $1 \mathrm{~h}$. As seen above, this excellent film stability is due to the drastic enhancement of both thermal and structural properties afforded by the triptycene moieties. Normalized electroluminescence (EL) spectra collected from two different devices are shown in Fig. 13.

The EL spectrum an OLED with the simple architecture ITO/ PEDOT-PSS/NPB/Tp-DMTPS/Ca displays a maximum at $516 \mathrm{~nm}$, which is not affected by the applied voltage (Fig. S20, ESI $\ddagger$ ). The insertion of $\mathrm{a} \mathrm{MoO}_{3}$ layer between PEDOT-PSS and NPB layers (ITO/PEDOT-PSS/ $\mathrm{MoO}_{3} / \mathrm{NPB} / \mathrm{Tp}$-DMTPS/Ca) induces a blue shift of $c a .20 \mathrm{~nm}$ in the EL spectrum. This could be the consequence

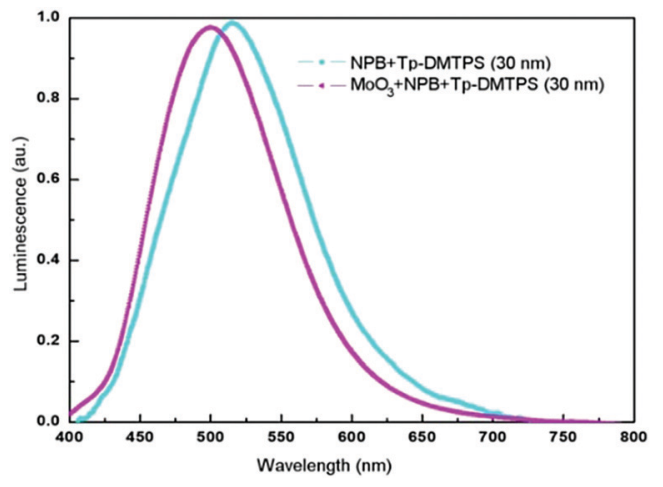

Fig. 13 Normalized EL spectra of Tp-DMTPS.

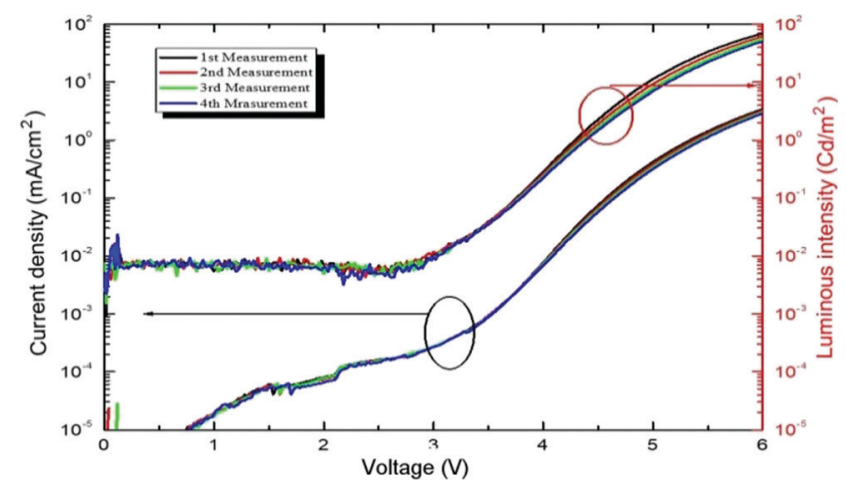

Fig. $14 J-V$ characteristics and luminous intensity versus voltage of an ITO/PEDOT-PSS/MoO $/$ /NPB/Tp-DMTPS/Ca device.

of a micro cavity effect, the emission wavelength being affected by the thickness of the active layer. ${ }^{64}$

The current density and luminuous intensity are shown in Fig. 14 for an ITO/PEDOT-PSS/ $\mathrm{MoO}_{3} / \mathrm{NPB} / \mathrm{Tp}$-DMTPS/Ca device. The stability and repeatability for these devices are quite good, the quantum efficiency which is $2.8 \mathrm{~cd} \mathrm{~A}^{-1}$ at $6.0 \mathrm{~V}$ is 35 fold higher than with DMTPS and the CIE color coordinates are $x=$ 0.228 and $y=0.412$ (Fig. S21, ESI $\$$ ). As anticipated from its lower solid state quantum yield, OLEDs with an ITO/PEDOT-PSS/NPB/ Tp-MPTPS/Ca architecture show lower performances than those obtained with Tp-DMTPS, i.e., $0.8 \mathrm{~cd} \mathrm{~A}^{-1}$ with CIE color coordinates of $x=0.301$ and $y=0.312$ (Fig. S22 and S23, ESI $\ddagger$ ).

\section{Electronic structure calculations}

The HOMO and LUMO energy levels of both optimized, $R$ - and $L$-conformers of Tp-DMTPS and Tp-MPTPS were estimated at the density functional theory (DFT) level using the B3LYP functional and the $6-31 G^{*}$ basis set. Calculations have been done on single molecules, either fully unconstrained, referred here as "optimized structures" or on structures for which the torsion angles have been frozen to their crystal values during the optimization, noted here as "constrained structures". The optimized structures and the shape of the HOMO and LUMO orbitals of Tp-DMTPS and Tp-MPTPS are depicted in Fig. 15.

As previously observed, the four phenyl rings decorating the central silole ring adopt a propeller-like arrangement. 


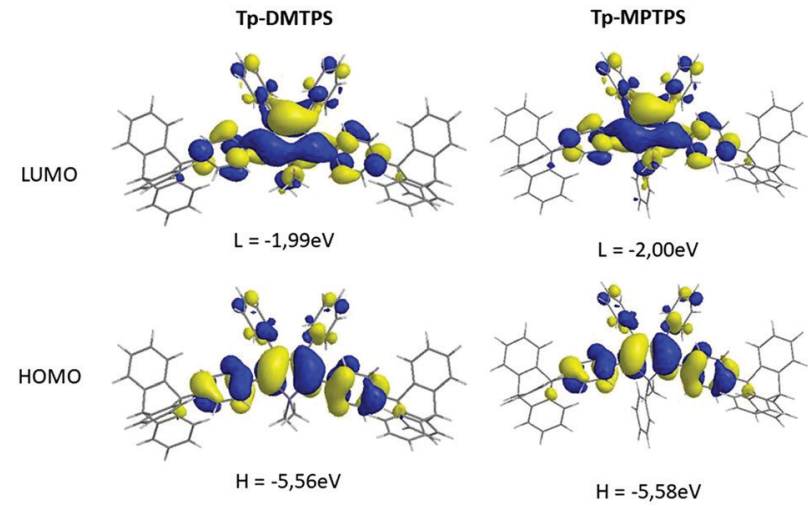

Fig. 15 Energy and shape of the HOMO and LUMO orbitals of optimized structures of Tp-DMTPS and Tp-MPTPS, as calculated at the B3LYP/6$31+G^{*}$ level.

Surprisingly, the calculations took rather well into account the strong $\mathrm{CH} / \pi$ interactions found in the crystal structures of both siloles with calculated bending angles of $c a .11^{\circ}$ (Tp-DMTPS) and $15^{\circ}$ (Tp-MPTPS) between the threefold axis of the triptycene group and the adjacent phenyl moiety. The HOMO orbitals of both derivatives are mainly located on the central silole core and phenyl rings at 2,5-positions while the substituents located at the 3 and 4-positions bear much weaker contributions. Calculated values are -5.56 and $-5.58 \mathrm{eV}$ for Tp-DMTPS and Tp-MPTPS, respectively, values that are close to the ones estimated from CV measurements (see Table 2). This indicates that the optimized structures are certainly close to the ones present in solution. For the LUMO orbitals, a significant $\sigma^{*}$-type orbital density is found on the two exocyclic Si-C bonds. This is typical for siloles and the resulting $\sigma\left(\mathrm{Si}-\mathrm{C}_{\text {exocyclic }}{ }^{*}-\pi(\text { butadiene })^{*}\right.$ hyperconjugation is responsible for the stabilization of the LUMO level; making those compounds good candidates for optoelectronic applications. ${ }^{7}$ The triptycene moieties are not involved in the frontier orbitals; Tp-DMTPS and Tp-MPTPS being therefore similar from an electronic point of view to their DMTPS and MPTPS parents.

Concerning $R$ - and $L$-conformers of Tp-DMTPS (Fig. 16), as expected from the increased values of the torsion angles with respect to fully optimized structures (Table 1), both conformers
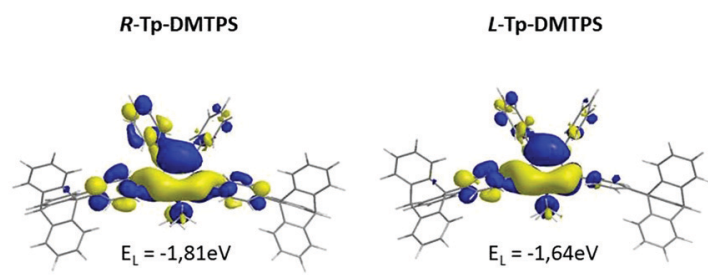

HOMO
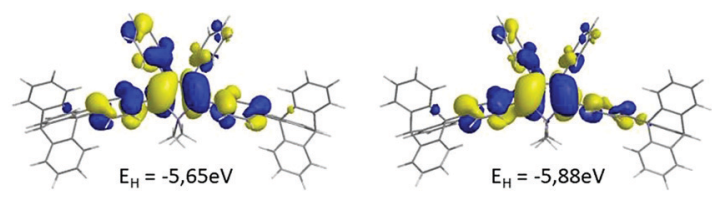

Fig. 16 Energy and shape of the HOMO and LUMO levels of constrained $R$ - and $L$-conformers of Tp-DMTPS as calculated at the B3LYP/6-31+G* level. display larger $E_{\mathrm{g}}$ values than for the optimized structure. Indeed, the $L$-conformer that is the less conjugated, displays the largest $E_{\mathrm{g}}$ value of $4.24 \mathrm{eV}$. Consequently, the emission of Tp-DMTPS is expected to be blue-shifted when passing from solution (calculated $E_{\mathrm{g}}=3.37 \mathrm{eV}$ ) to the solid state, in contrast to experimental observations. This clearly suggests that the redshift observed experimentally does not stem from the intrinsic properties of the molecules, but rather from the strong $\mathrm{CH} / \pi$ intermolecular interactions that occur in the solid. ${ }^{65,66}$

\section{Charge transport calculations}

Finally, we decided to perform quantum-chemical calculation to evaluate both the role the $\mathrm{CH} / \pi$ intermolecular interactions and the conformational modifications in a supramolecular property such as charge transport. Therefore, the estimation of the hole and electron mobilities for both $R$ - and $L$-conformers of Tp-DMTPS as well as DMTPS as a reference were carried out. ${ }^{67,68}$ Interestingly, our methodology reproduces nicely the measured electrontransporting character of DMTPS ${ }^{47}$ with the highest calculated electron mobilities of $0.134 \mathrm{~cm}^{2}(\mathrm{~V} \mathrm{~s})^{-1}$, compared to $0.038 \mathrm{~cm}^{2}$ $(\mathrm{V} \mathrm{s})^{-1}$ for holes (see Fig. S24, ESI $\$$ ). In contrast to DMTPS for which high transport properties are almost isotropic, Tp-DMTPS only exhibits a two-dimensional charge transport character within the $b c$ plane, especially along the $b$ axes. It is worthy to note that this is also in this direction that the main $\mathrm{CH} / \pi$ intermolecular interactions are running (see above). The twodimensional behavior is a result of the 'insulating character' of the triptycene moiety: since the HOMO and LUMO orbitals are localized on the TPS subunit and not on the triptycene, hole and electron hopping events are highly unlikely along the triptycene direction. Surprisingly, our simulations reveal that, compared to

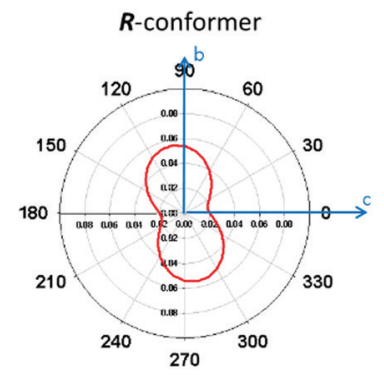

$\mu_{\text {max, holes }}=0.055 \mathrm{~cm}^{2} / \mathrm{Vs}$

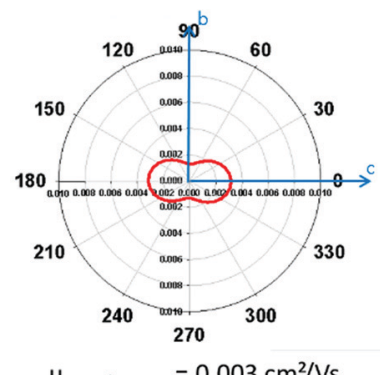

$\mu_{\text {max,electrons }}=0.003 \mathrm{~cm}^{2} / \mathrm{Vs}$

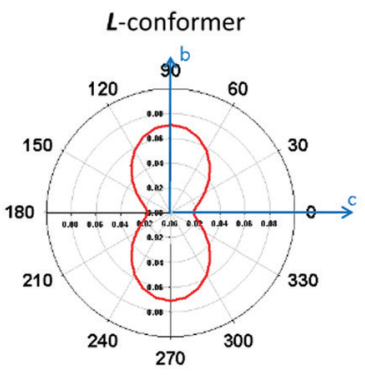

$\mu_{\text {max, holes }}=0.071 \mathrm{~cm}^{2} / \mathrm{Vs}$

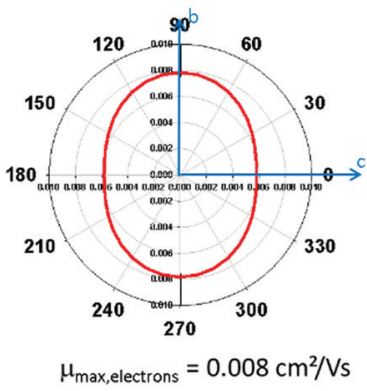

Fig. 17 Anisotropy of the hole (top) and electron (bottom) mobilities (in $\mathrm{cm}^{2}(\mathrm{~V} \mathrm{~s})^{-1}$ ) in the $R$ - (left) and $L$ - (right) conformers of Tp-DMTPS. The horizontal [vertical] direction corresponds to the $c$-[b-] unit cell axis. Note that the mobility scale is different for holes and electrons. 
DMTPS, hole mobilities are slightly enhanced while electron mobilities are significantly lowered, down to values never exceeding $0.008 \mathrm{~cm}^{2}(\mathrm{~V} \mathrm{~s})^{-1}$, see Fig. 17. Moreover, an increase of the mobilities is observed when passing from the $R$ - to the $L$-conformer of Tp-DMTPS. This result is in good agreement with the conclusion drawn by the Hirshfeld surface analysis (see Fig. 5), indicating that the $L$-conformer display stronger $\mathrm{CH} / \pi$ intermolecular interactions than the $R$-conformer. These calculations also show that these interactions are likely involved in the charge transport pathways.

Grafting a triptycene fragment on a TPS central core therefore strongly modifies its charge transport properties by (i) reducing its charge transfer character from 3-D to 2-D and by (ii) inducing a stronger hole-transporting character while keeping a moderate electron mobility. These results imply that Tp-DMTPS is able to transport both types of charge carriers reasonably well, consistent with the device performances described above.

\section{Conclusions}

Experimental and theoretical studies of two silole-triptycene derivatives Tp-DMTPS and Tp-MPTPS are presented. Both compounds display a very good AIE activity as well as increased thermal and structural stabilities in comparison with other silole congeners. Consequently, a substantial improvement is found in the formation of stable, amorphous thin films as well as in the performance of simple OLED. Further improvement of the devices performance should be reached through the correction of the charge-transport balance by molecular engineering. ${ }^{6,69}$ Among the two molecules, Tp-DMTPS displays a very interesting photophysical behavior. In contrast to what is frequently observed, an appreciable red-shift is observed when passing from either the solution or the tiny aggregates to the crystals. Moreover a pronounced reversible mechanohypsochromic phenomenon with a color emission changing from yellow-orange to bluegreen is observed when microcrystalline powders are ground. Based on single crystal structural analysis, ${ }^{13} \mathrm{C}$ CP-MAS NMR and theoretical calculations this behavior was rationalized. We have demonstrated the significance of both molecular conformation and intermolecular $\mathrm{CH} / \pi$ interactions. ${ }^{13} \mathrm{C}$ CP-MAS NMR experiments conducted either on microcrystalline or ground powders confirmed that despite of the loss of crystallinity upon grinding, strong intermolecular $\mathrm{CH} / \pi$ interactions are preserved. This reflects the strong structure-directing properties brought by the triptycene groups. The origin of the red-shift observed when passing from isolated molecules to the crystal may be explained by the stabilization of the frontiers energy levels through $\mathrm{CH} / \pi$ intermolecular interactions. On the other hand, the origin of the mechanohypsochromism could be explained by the fact that the more energetic conformer is favored when the crystal environmental strain is diminished. Our results therefore indicate that the attachment of triptycene moieties to $\pi$-conjugated molecules is an interesting way to improve their thermal, structural and photophysical properties for sensing or optoelectronic applications. However, further experiments and sophisticated calculations are needed to fully understand this very original behavior.

\section{Conflicts of interest}

There are no conflicts to declare.

\section{Acknowledgements}

The authors thank the CNRS and the Universite of Montpellier for financial support. The authors are also grateful to the Agence Nationale de la Recherche (ANR) for financial support of the research project PSICO (\# ANR-07-BLAN-0281-01). The authors also thank technical staff of the Plateforme d'Analyses et de Caracterisations (PAC) de l'Institut Charles Gerhardt de Montpellier (ICGM) for their help and fruitful discussions. The work in Mons was supported by the European Commission and Region Wallonne FEDER program (BIORGEL project), and by the FNRS-FRFC (Consortium des Équipements de Calcul Intensif - CÉCI). P. Gerbier and S. Clément are grateful to Mr Mehdi Mokhtari for his experimental work during his DUT internship.

\section{Notes and references}

1 J. Dubac, A. Laporterie and G. Manuel, Group 14 metalloles. 1. Synthesis, organic chemistry, and physicochemical data, Chem. Rev., 1990, 90, 215-263.

2 S. Yamaguchi, R.-Z. Jin, K. Tamao and M. Shiro, Synthesis of a Series of 1,1-Difunctionalized Siloles, Organometallics, 1997, 16, 2230-2232.

3 S. Yamaguchi and K. Tamao, Silole-containing $\sigma$ - and $\pi$-conjugated compounds, J. Chem. Soc., Dalton Trans., 1998, 3693-3702.

4 K. Tamao, M. Uchida, T. Izumizawa, K. Furukawa and S. Yamaguchi, Silole Derivatives as Efficient Electron Transporting Materials, J. Am. Chem. Soc., 1996, 118, 11974-11975.

5 S. Yamaguchi, T. Endo, M. Uchida, T. Izumizawa, K. Furukawa and K. Tamao, Toward new materials for organic electroluminescent devices: synthesis, structures, and properties of a series of 2,5-diaryl-3,4-diphenylsiloles, Chem. - Eur. J., 2000, 6, 1683-1692.

6 L. Aubouy, P. Gerbier, N. Huby, G. Wantz, L. Vignau, L. Hirsch and J.-M. Janot, Synthesis of new dipyridylphenylaminosiloles for highly emissive organic electroluminescent devices, New J. Chem., 2004, 28, 1086-1090.

7 Y. Cai, A. Qin and B. Z. Tang, Siloles in optoelectronic devices, J. Mater. Chem. C, 2017, 5, 7375-7389.

8 K. Amro, A. K. Thakur, J. Rault-Berthelot, C. Poriel, L. Hirsch, W. E. Douglas, S. Clement and P. Gerbier, 2,5-Thiophene substituted spirobisiloles - synthesis, characterization, electrochemical properties and performance in bulk heterojunction solar cells, New J. Chem., 2013, 37, 464-473.

9 J. Luo, Z. Xie, J. W. Y. Lam, L. Cheng, H. Chen, C. Qiu, H. S. Kwok, X. Zhan, Y. Liu, D. Zhu and B. Z. Tang, Aggregationinduced emission of 1-methyl-1,2,3,4,5-pentaphenylsilole, Chem. Commun., 2001, 1740-1741.

10 G. Feng and B. Liu, Aggregation-induced emission (AIE) dots: Emerging theranostic nanolights, Acc. Chem. Res., 2018, 51, 1404-1414. 
11 D. Wang and B. Z. Tang, Aggregation-Induced Emission Luminogens for Activity-Based Sensing, Acc. Chem. Res., 2019, 52, 2559-2570.

12 Z. Zhao, B. He and B. Z. Tang, Aggregation-induced emission of siloles, Chem. Sci., 2015, 6, 5347-5365.

13 M. Arribat, E. Remond, S. Richeter, P. Gerbier, S. Clement and F. Cavelier, Silole amino acids with aggregation-induced emission features synthesized by hydrosilylation, Eur. J. Org. Chem., 2019, 2275-2281.

14 J. Mei, N. L. C. Leung, R. T. K. Kwok, J. W. Y. Lam and B. Z. Tang, Aggregation-Induced Emission: Together We Shine, United We Soar!, Chem. Rev., 2015, 115, 11718-11940.

15 Y. Xie and Z. Li, The development of mechanoluminescence from organic compounds: breakthrough and deep insight, Mater. Chem. Front., 2020, 4, 317-331.

16 Z. Chi, X. Zhang, B. Xu, X. Zhou, C. Ma, Y. Zhang, S. Liu and $\mathrm{J}$. Xu, Recent advances in organic mechanofluorochromic materials, Chem. Soc. Rev., 2012, 41, 3878-3896.

17 J.-Y. Zhu, C.-X. Li, P.-Z. Chen, Z. Ma, B. Zou, L.-Y. Niu, G. Cui and Q.-Z. Yang, A polymorphic fluorescent material with strong solid state emission and multi-stimuli-responsive properties, Mater. Chem. Front., 2020, 4, 176-181.

18 L. Wilbraham, M. Louis, D. Alberga, A. Brosseau, R. Guillot, F. Ito, F. Labat, R. Metivier, C. Allain and I. Ciofini, Revealing the Origins of Mechanically Induced Fluorescence Changes in Organic Molecular Crystals, Adv. Mater., 2018, 30, 1800817.

19 X. Luo, J. Li, C. Li, L. Heng, Y. Q. Dong, Z. Liu, Z. Bo and B. Z. Tang, Reversible Switching of the Emission of Diphenyldibenzofulvenes by Thermal and Mechanical Stimuli, Adv. Mater., 2011, 23, 3261-3265.

20 N. Zhao, Z. Yang, J. W. Y. Lam, H. H. Y. Sung, N. Xie, S. Chen, H. Su, M. Gao, I. D. Williams, K. S. Wong and B. Z. Tang, Benzothiazolium-functionalized tetraphenylethene: an AIE luminogen with tunable solid-state emission, Chem. Commun., 2012, 48, 8637-8639.

21 K. Walzer, B. Maennig, M. Pfeiffer and K. Leo, Highly Efficient Organic Devices Based on Electrically Doped Transport Layers, Chem. Rev., 2007, 107, 1233-1271.

22 P. Kordt, J. J. M. van der Holst, M. Al Helwi, W. Kowalsky, F. May, A. Badinski, C. Lennartz and D. Andrienko, Modeling of Organic Light Emitting Diodes: From Molecular to Device Properties, Adv. Funct. Mater., 2015, 25, 1955-1971.

23 X. Zhou, J. He, L. S. Liao, M. Lu, X. M. Ding, X. Y. Hou, X. M. Zhang, X. Q. He and S. T. Lee, Real-time observation of temperature rise and thermal breakdown processes in organic LEDs using an IR imaging and analysis system, Adv. Mater., 2000, 12, 265-269.

24 G. Wantz, L. Derue, O. Dautel, A. Rivaton, P. Hudhomme and C. Dagron-Lartigau, Stabilizing polymer-based bulk heterojunction solar cells via crosslinking, Polym. Int., 2014, 63, 1346-1361.

25 T. M. Swager, Iptycenes in the Design of High Performance Polymers, Acc. Chem. Res., 2008, 41, 1181-1189.

$26 \mathrm{~J}$. H. Chong and M. J. MacLachlan, Iptycenes in supramolecular and materials chemistry, Chem. Soc. Rev., 2009, 38, 3301-3315.
27 Y. Jiang and C.-F. Chen, Recent Developments in Synthesis and Applications of Triptycene and Pentiptycene Derivatives, Eur. J. Org. Chem., 2011, 6377-6403.

28 G. M. Locke, S. S. R. Bernhard and M. O. Senge, Nonconjugated Hydrocarbons as Rigid-Linear Motifs: Isosteres for Material Sciences and Bioorganic and Medicinal Chemistry, Chem. Eur. J., 2019, 25, 4590-4647.

29 H.-H. Chou, H.-H. Shih and C.-H. Cheng, Triptycene derivatives as high- $T_{\mathrm{g}}$ host materials for various electrophosphorescent devices, J. Mater. Chem., 2010, 20, 798-805.

30 S. Jhulki, T. J. Chow and J. N. Moorthy, Benzophenoneimbedded benzoyltriptycene with high triplet energy for application as a universal host material in phosphorescent organic light-emitting diodes (PhOLEDs), New J. Chem., 2016, 40, 6854-6859.

31 Y. Wang, Y.-P. Xiao, Y.-Y. Zhou, C.-G. Hu, B.-H. Tong, S.-H. Ye and Q.-B. Mei, Novel phosphorescent triptycenebased Ir(III) complexes for organic light-emitting diodes, Dalton Trans., 2019, 48, 16289-16297.

32 K. Nagura, S. Saito, H. Yusa, H. Yamawaki, H. Fujihisa, H. Sato, Y. Shimoikeda and S. Yamaguchi, Distinct Responses to Mechanical Grinding and Hydrostatic Pressure in Luminescent Chromism of Tetrathiazolylthiophene, J. Am. Chem. Soc., 2013, 135, 10322-10325.

33 M. Tanioka, S. Kamino, A. Muranaka, Y. Ooyama, H. Ota, Y. Shirasaki, J. Horigome, M. Ueda, M. Uchiyama, D. Sawada and S. Enomoto, Reversible Near-Infrared/Blue Mechanofluorochromism of Aminobenzopyranoxanthene, J. Am. Chem. Soc., 2015, 137, 6436-6439.

34 L. Wang, K. Wang, B. Zou, K. Ye, H. Zhang and Y. Wang, Luminescent Chromism of Boron Diketonate Crystals: Distinct Responses to Different Stresses, Adv. Mater., 2015, 27, 2918-2922.

35 J. Wu, Y. Cheng, J. Lan, D. Wu, S. Qian, L. Yan, Z. He, X. Li, K. Wang, B. Zou and J. You, Molecular Engineering of Mechanochromic Materials by Programmed C-H Arylation: Making a Counterpoint in the Chromism Trend, J. Am. Chem. Soc., 2016, 138, 12803-12812.

36 S. Ying, M. Chen, Z. Liu, M. Zheng, H. Zhang, S. Xue and W. Yang, Unusual mechanohypsochromic luminescence and unique bidirectional thermofluorochromism of longalkylated simple DPP dyes, J. Mater. Chem. C, 2017, 5, 5994-5998.

37 K. Zhang, Z. Zhang, X. Fan, L. Tang, Q. Ding, K. Yang, M. Zhai, H. Zhang, S. Xue and W. Yang, Enabling DPP derivatives to show multistate emission and developing the multifunctional materials by rational branching effect, Dyes Pigm., 2018, 159, 290-297.

38 M. Uchida, T. Izumizawa, T. Nakano, S. Yamaguchi, K. Tamao and K. Furukawa, Structural Optimization of 2,5-Diarylsiloles as Excellent Electron-Transporting Materials for Organic Electroluminescent Devices, Chem. Mater., 2001, 13, 2680-2683.

39 S. Murphy, X. Yang and G. B. Schuster, Cyanine Borate Salts that Form Penetrated Ion Pairs in Benzene Solution: Synthesis, Properties, and Structure, J. Org. Chem., 1995, 60, 2411-2422. 
$40 \mathrm{M}$. Nishio, The $\mathrm{CH} / \pi$ hydrogen bond in chemistry. Conformation, supramolecules, optical resolution and interactions involving carbohydrates, Phys. Chem. Chem. Phys., 2011, 13, 13873-13900.

41 K. Nikitin, C. Fleming, H. Mueller-Bunz, Y. Ortin and M. J. McGlinchey, Severe Energy Costs of Double Steric Interactions: towards a Molecular Clamp, Eur. J. Org. Chem., 2010, 5203-5216.

42 L. J. Sicard, H.-C. Li, Q. Wang, X.-Y. Liu, O. Jeannin, J. RaultBerthelot, L.-S. Liao, Z.-Q. Jiang and C. Poriel, C1-Linked Spirobifluorene Dimers: Pure Hydrocarbon Hosts for HighPerformance Blue Phosphorescent OLEDs, Angew. Chem., Int. Ed., 2019, 58, 3848-3853.

43 Y. N. Imai, Y. Inoue, I. Nakanishi and K. Kitaura, Cl- $\pi$ interactions in protein-ligand complexes, Protein Sci., 2008, 17, 1129-1137.

44 M. A. Spackman and P. G. Byrom, A novel definition of a molecule in a crystal, Chem. Phys. Lett., 1997, 267, 215-220.

45 S. K. Wolff, D. J. Grimwood, J. J. McKinnon, M. J. Turner, D. Jayatilaka and M. A. Spackmann, CrystalExplorer, University of Western Australia, 2012.

46 J. J. McKinnon, D. Jayatilaka and M. A. Spackman, Towards quantitative analysis of intermolecular interactions with Hirshfeld surfaces, Chem. Commun., 2007, 3814-3816.

47 G. Yu, S. Yin, Y. Liu, J. Chen, X. Xu, X. Sun, D. Ma, X. Zhan, Q. Peng, Z. Shuai, B. Tang, D. Zhu, W. Fang and Y. Luo, Structures, Electronic States, Photoluminescence, and Carrier Transport Properties of 1,1-Disubstituted 2,3,4,5-Tetraphenylsiloles, J. Am. Chem. Soc., 2005, 127, 6335-6346.

48 S. Yamaguchi and K. Tamao, Silole-containing $\pi$-conjugated polymers. 5. Theoretical study of the electronic structure of 2,2'-bisilole in comparison with 1,1'-bi-1,3-cyclopentadiene: $\sigma^{*}-\pi^{*}$ conjugation and a low-lying LUMO as the origin of the unusual optical properties of $3,3^{\prime}, 4,4^{\prime}$-tetraphenyl-2,2'-bisilole, Bull. Chem. Soc. Jpn., 1996, 69, 2327-2334.

49 J. Chen, C. C. W. Law, J. W. Y. Lam, Y. Dong, S. M. F. Lo, I. D. Williams, D. Zhu and B. Z. Tang, Synthesis, Light Emission, Nanoaggregation, and Restricted Intramolecular Rotation of 1,1-Substituted 2,3,4,5-Tetraphenylsiloles, Chem. Mater., 2003, 15, 1535-1546.

50 Y. Hong, J. W. Y. Lam and B. Z. Tang, Aggregation-induced emission: phenomenon, mechanism and applications, Chem. Commun., 2009, 4332-4353.

51 R. Cao, L. Ai, H. Yang, S. Li and C. Xu, Aggregation-tuned dual emission of silole derivatives: synthesis, crystal structure, and photophysical properties, New J. Chem., 2020, 44, 5049-5055.

52 L. Heng, J. Zhai, A. Qin, Y. Zhang, Y. Dong, B. Z. Tang and L. Jiang, Fabrication of hexaphenylsilole nanowires and their morphology-tunable photoluminescence, ChemPhysChem, 2007, 8, 1513-1518.

53 J. Yang, N. Sun, J. Huang, Q. Li, Q. Peng, X. Tang, Y. Dong, D. Ma and Z. Li, New AIEgens containing tetraphenylethene and silole moieties: tunable intramolecular conjugation, aggregation-induced emission characteristics and good device performance, J. Mater. Chem. C, 2015, 3, 2624-2631.
54 C. Wang, B. Xu, M. Li, Z. Chi, Y. Xie, Q. Li and Z. Li, A stable tetraphenylethene derivative: aggregation-induced emission, different crystalline polymorphs, and totally different mechanoluminescence properties, Mater. Horiz., 2016, 3, 220-225.

55 Y. Dong, J. W. Y. Lam, Z. Li, A. Qin, H. Tong, Y. Dong, X. Feng and B. Z. Tang, Vapochromism of Hexaphenylsilole, J. Inorg. Organomet. Polym. Mater., 2005, 15, 287-291.

56 T. Mutai, H. Tomoda, T. Ohkawa, Y. Yabe and K. Araki, Switching of polymorph-dependent ESIPT luminescence of an imidazo[1,2-a]pyridine derivative, Angew. Chem., Int. Ed., 2008, 47, 9522-9524.

57 Y. Sagara and T. Kato, Mechanically induced luminescence changes in molecular assemblies, Nat. Chem., 2009, 1, 605-610.

58 J. Yang, Z. Ren, B. Chen, M. Fang, Z. Zhao, B. Z. Tang, Q. Peng and Z. Li, Three polymorphs of one luminogen: how the molecular packing affects the RTP and AIE properties?, J. Mater. Chem. C, 2017, 5, 9242-9246.

59 A. P. Kulkarni, C. J. Tonzola, A. Babel and S. A. Jenekhe, Electron Transport Materials for Organic Light-Emitting Diodes, Chem. Mater., 2004, 16, 4556-4573.

60 C. M. Cardona, W. Li, A. E. Kaifer, D. Stockdale and G. C. Bazan, Electrochemical Considerations for Determining Absolute Frontier Orbital Energy Levels of Conjugated Polymers for Solar Cell Applications, Adv. Mater., 2011, 23, 2367-2371.

61 C. Paul-Roth, J. Rault-Berthelot, G. Simonneaux, C. Poriel, M. Abdalilah and J. Letessier, Electroactive films of poly(tetraphenylporphyrins) with reduced bandgap, J. Electroanal. Chem., 2006, 597, 19-27.

62 N. Huby, L. Hirsch, G. Wantz, L. Vignau, A. S. Barriere, J. P. Parneix, L. Aubouy and P. Gerbier, Injection and transport processes in organic light emitting diodes based on a silole derivative, J. Appl. Phys., 2006, 99, 084907.

63 B. Z. Tang, X. Zhan, G. Yu, P. P. S. Lee, Y. Liu and D. Zhu, Efficient blue emission from siloles, J. Mater. Chem., 2001, 11, 2974-2978.

64 A. Dodabalapur, L. J. Rothberg, T. M. Miller and E. W. Kwock, Microcavity effects in organic semiconductors, Appl. Phys. Lett., 1994, 64, 2486-2488.

65 N. Huby, L. Hirsch, L. Aubouy, P. Gerbier, A. Van Der Lee, F. Amy and A. Kahn, Evidence of environmental strains on charge injection in silole-based organic light-emitting diodes, Phys. Rev. B: Condens. Matter Mater. Phys., 2007, 75, 115416.

66 D. Kokkin, M. Ivanov, J. Loman, J.-Z. Cai, B. Uhler, N. Reilly, R. Rathore and S. A. Reid, $\pi-\pi$ stacking $v s$. C-H/ $\pi$ interaction: Excimer formation and charge resonance stabilization in van der Waals clusters of 9,9'-dimethylfluorene, J. Chem. Phys., 2018, 149, 134314.

67 C. Niebel, Y. Kim, C. Ruzie, J. Karpinska, B. Chattopadhyay, G. Schweicher, A. Richard, V. Lemaur, Y. Olivier, J. Cornil, A. R. Kennedy, Y. Diao, W.-Y. Lee, S. Mannsfeld, Z. Bao and Y. H. Geerts, Thienoacene dimers based on the thieno[3,2-b]thiophene moiety: synthesis, characterization 
and electronic properties, J. Mater. Chem. C, 2015, 3, 674-685.

68 G. Schweicher, V. Lemaur, C. Niebel, C. Ruzie, Y. Diao, O. Goto, W.-Y. Lee, Y. Kim, J.-B. Arlin, J. Karpinska, A. R. Kennedy, S. R. Parkin, Y. Olivier, S. C. B. Mannsfeld, J. Cornil, Y. H. Geerts and Z. Bao, Bulky end-capped [1]benzothieno[3,2-b]benzothiophenes: Reaching high-mobility organic semiconductors by fine tuning of the crystalline solid-state order, Adv. Mater., 2015, 27, 3066-3072.

69 L. Chen, Y. Jiang, H. Nie, P. Lu, H. H. Y. Sung, I. D. Williams, H. S. Kwok, F. Huang, A. Qin, Z. Zhao and B. Z. Tang, Creation of Bifunctional Materials: Improve Electron-Transporting Ability of Light Emitters Based on AIE-Active 2,3,4,5-Tetraphenylsiloles, Adv. Funct. Mater., 2014, 24, 3621-3630. 